\title{
Vortex Pair of Coaxial Helical Filaments
}

\section{Okulov, V. L.}

\section{Published in:}

Journal of Applied Mechanics and Technical Physics

Link to article, DOI:

$10.1134 /$ S0021894420030049

Publication date:

2020

Document Version

Peer reviewed version

Link back to DTU Orbit

\section{Citation (APA):}

Okulov, V. L. (2020). Vortex Pair of Coaxial Helical Filaments. Journal of Applied Mechanics and Technical Physics, 61(3), 343-349. https://doi.org/10.1134/S0021894420030049

\section{General rights}

Copyright and moral rights for the publications made accessible in the public portal are retained by the authors and/or other copyright owners and it is a condition of accessing publications that users recognise and abide by the legal requirements associated with these rights.

- Users may download and print one copy of any publication from the public portal for the purpose of private study or research.

- You may not further distribute the material or use it for any profit-making activity or commercial gain

- You may freely distribute the URL identifying the publication in the public portal

If you believe that this document breaches copyright please contact us providing details, and we will remove access to the work immediately and investigate your claim 


\title{
VORTEX PAIR OF COAXIAL HELICAL FILAMENTS
}

\author{
V. L. Okulov
}

UDC 532.5.01

\begin{abstract}
Possible existence of vortex pairs of coaxially rotating helical filaments with circulation in the opposite directions is theoretically studied. Such pairs are generated, e.g., by a rotating blade of a rotor with identical intensities of the tip and shifted axial vortices. Conditions that ensure uniform rotation of the vortex pair of helical filaments are found, which is needed for modeling vortex wakes behind rotor blades.
\end{abstract}

Keywords: vortex dynamics, helical vortex, vortex pair, equilibrium rotation.

\section{DOI:}

\section{INTRODUCTION}

Alekseenko and Shtork [1] published an important experimental result that confirms the existence of an equilibrium state for two helical filaments of identical intensity. The problem of equilibrium states of vortices is the classical problem of hydrodynamics; however, only the plane case has been considered for a long time [2], and the problem was reduced to determining equilibrium configurations, i.e., vortex polygons whose vertices contain point vortices with identical circulation intensities in terms of magnitude and direction $[3,4]$. This problem was initiated by the experiment where identical configurations were observed for floating identical magnets. These configurations are similar to equilibrium polygons of point vortices, which had to be studied for developing the vortex model of the atom [5]. Similar to the classical experiment on equilibrium of magnets, which formed the basis of the theory of equilibrium states of point vortices, the experimental study of Alekseenko and Shtork [1] initiated a series of theoretical and numerical investigations aimed at determining stable equilibrium configurations of helical vortices [69]. Stability of a multiplet of helical vortices with identical circulation intensities located uniformly on a cylindrical surface, forming a regular vortex polygon, and uniformly moving along the axis was theoretically studied [6]. Then the possibility of existence of an equilibrium configuration for two helical vortices with identical circulation intensities and different radii asymmetrically arranged with respect to the common central axis (asymmetric helical vortex doublet) was theoretically confirmed [9]. The existence of such an asymmetric doublet in addition to the symmetric doublet described in [1] was not confirmed experimentally.

It should be noted that the classical formulation of problems of point vortices is not limited to the case of identical eirculation intensities; another case of interest is the case of vortices with identical eirculation intensities of the opposite signs. In the classical plane case, these two vortices are called the vortex pair; this term was introduced and described by Helmholtz earlier than the problem of vortex polygons. In [10], Helmholtz considered possible motions of a set of rectangular helical filaments or two-dimensional point vortices. In particular, it follows from [10] that there is only one unique solution for point vortices, where the vortex pair with identical, but oppositely directed circulations can move only in a straight-line manner, and point vortices can rotate only if they have different circulation intensities around their center of gravity, which is located outside the vortices on the line connecting

Novosibirsk State University, Novosibirsk, 630090 Russia. Wind Energy Department, Technical University of Denmark, DK-2800 Lyngby, Denmark; vaok@dtu.dk. Translated from Prikladnaya Mekhanika i Tekhnicheskaya Fizika, Vol. 61, No. 3, pp. 34-41, May-June, 2020. Original article submitted March 24, 2020; revision submitted March 24, 2020; accepted for publication March 30, 2020.

${ }^{*}$ Corresponding author. 
(a)

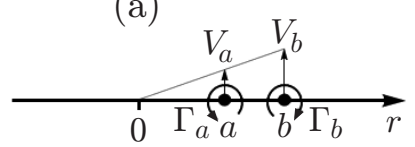

(b)

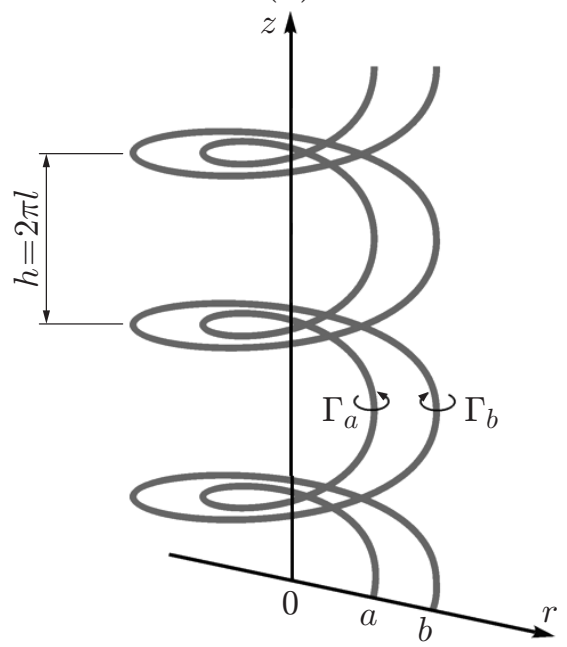

Fig. 1. Possible configurations of the vortex pair: (a) two point vortices; (b) two helical vortices.

them. However, generation of straight-line or point vortices in flows is a rare phenomenon: engineering and power engineering applications usually involve curved and helical vortices [11]. In particular, vortex pairs are generated in the near wake behind propeller or turbine blades and possess an ideal helical shape [12]; moreover, the helical shape is observed for both vortices, i.e., external (tip) and internal (sleeve) vortices shed from the tip and sleeve edges of the blade and having circulation intensities of different signs. In the case of a single rotating wing or blade for the NEZh blade model [13], a situation may occur where the shed external and internal vortices in the vortex pair have circulation intensities with identical magnitudes, but different signs [14].

In the case of a point analog of the vortex pair [10], uniform rotation is possible only for vortices of different intensities (Fig. 1a), while vortices with identical and oppositely directed circulations can move only a long a straight line, which contradicts the experimental data [15] where it was found that vortices shed from different edges of the wing should have close-in-value intensities, but different signs. In contrast to straight-line vortices, each helical vortex can also rotate as a whole [16]. This additional self-induced motion can change the dynamic behavior of the pair of helical vortices for which the solution becomes possible in the case of uniform rotation of both vortices around a common axis (Fig. 1b). In contrast to the above-described asymmetric doublet, which has been predicted only theoretically by now [9], the vortex pair where the helical filaments having circulation intensities of different signs rotate one inside the other has been observed only visually in the experiment [15]. This case has not been yet studied theoretically; therefore, the goal of the present investigation is to verify the possibility of uniform rotation of a helical vortex pair with and identical pitch and with the identical circulations of the opposite signs. Specific features of three-dimensional of thin helical vortex filaments in ideal fluids are considered; in addition of their interaction, we also study self-induced motion of vortex tubes in the binormal direction away from their axis, which follows from the equations of motion of a three-dimensional vortex filament derived at the beginning of the 20th century when the concept of self-induced motion of vortex filaments was put forward [13, 17]. In contrast to various approximate models, the present study is based on the Kawada-Hardin solution [18], in which specific features for an infinite thin helical vortex filament were identified and which was applied for determining self-induced velocities [16] summed up with the velocity of induction of the other vortex filament. 
(a)

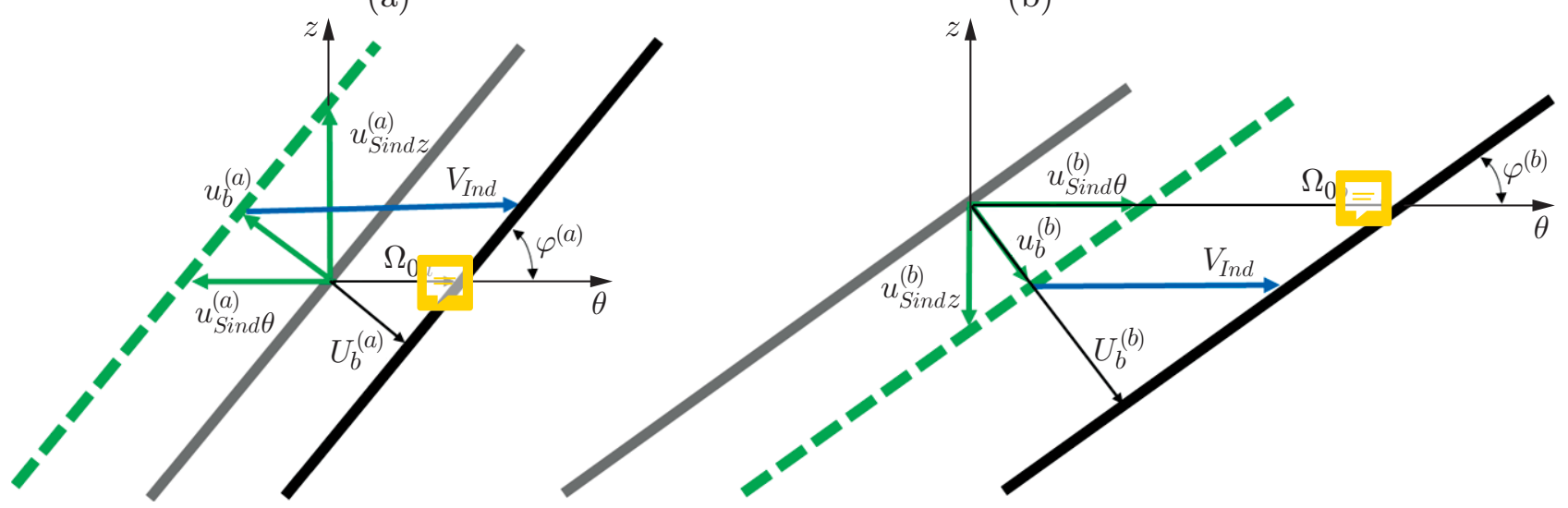

(b)

Fig. 2. Rectangles of velocities for cylindrical evolvents of the internal (a) and external (b) helical vortices.

\section{EQUATIONS OF MOTION OF THE HELICAL VORTEX PAIR}

To determine the equilibrium conditions for the helical vortex pair (see Fig. 1b), we consider two thin helical vortices located on two support cylinders with fixed radii $a$ and $b$ and one common geometric pitch $h$ (or $l=h /(2 \pi)$ ). This corresponds to two dimensionless steps $\tau_{a}=l / a, \tau_{b}=l / b$ for each filament. Let us study arbitrary absolute values of the vortex circulation intensities $\Gamma_{a}$ and $\Gamma_{b}$. This is important for considering the limiting case of a rotating pair of straight-line vortices $(h=\infty)$ at $\Gamma_{a} / \Gamma_{b}=-b / a$. For finite values $h=2 \pi l$, possible equilibrium rotation at $\Gamma_{a}=-\Gamma_{b}$ is determined by varying the problem parameters. Then these limiting cases are studied because it is impossible to predict that vortex rolling into the helical shape with a decrease in the total pitch to a certain finite value $h=h^{*}$ can lead to equilibrium rotation with identical absolute values of circulation intensity $\Gamma_{a}=-\Gamma_{b}$. Let us also assume that both vortices have circular cross sections with identical small radii $\varepsilon \ll h$ and $\varepsilon \ll b$. Both helical vortices in the pair represent a superposition of helical vortex filaments aligned at a certain time instant parallel to their axes, and vorticity is uniformly distributed in the vortex core cross section [16].

To study the equilibrium states of the pair of helical vortices under consideration (see Fig. 1b), we determine the angular velocity of each vortex. In contrast to the Helmholtz straight-line filaments (or point vortices) whose motion is determined by the velocity induced only by the other vortex of the pair at the point of calculated vortex location, the common angular velocity for each helical vortex around their common center consists of two components

$$
\Omega^{(a)}=\Omega_{\text {Ind }}^{(a)}+\Omega_{\text {Sind }}^{(a)}, \quad \Omega^{(b)}=\Omega_{\text {Ind }}^{(b)}+\Omega_{\text {Sind }}^{(b)},
$$

where $\Omega_{\text {Ind }}^{(a)}=V_{\text {Ind }}^{(b)}(a) / a$ and $\Omega_{\text {Ind }}^{(b)}=V_{\text {Ind }}^{(a)}(b) / b$ are the angular velocities at the vortex position points $a$ or $b$ induced by the other vortex from the position $b$ or $a$, respectively (see Fig. 1 ); $\Omega_{\text {Sind }}^{(a)}$ and $\Omega_{\text {Sind }}^{(b)}$ are the velocities of self-induced motion of the vortex itself [16] calculated at the point $a$ or $b$. The notion of angular velocities for helical vortices moving in the binormal direction should be also specified. This problem has not been considered earlier, and the motion of helical vortices was erroneously identified with the motion of fluid particles [19]. To avoid wrong interpretations, we construct velocity rectangles for each vortex of the pair in a plane cylindrical evolvent of both support cylinders (Fig. 2).

Fixing the cross section $z=0$ in a motionless polar coordinate system and neglecting the influence of the finite size of the other vortex core on the vortex under consideration, we find the velocities of circumferential motion of the vortices in this fixed cross section. They do not coincide with the projections of the binormal velocity onto the tangential direction because of additional displacement of the helical vortex during its translational motion. Following $[13,16]$, from the velocity triangles in Fig. 2, we obtain the self-induced component

$$
\Omega_{\text {Sind }}^{(a)} a=-u_{b}(a) / \sin \varphi^{(a)}, \quad \Omega_{\text {Sind }}^{(b)} b=-u_{b}(b) / \sin \varphi^{(b)},
$$

where $\sin \varphi^{(x)}=x / \sqrt{l^{2}+x^{2}}$. 
Determination of the binormal component $u_{b}(x)$ of self-induced motion is a complicated problem whose solution was a challenge for many researchers (see [13, 20-23], etc.). The general solution for a helical vortex with a small cross section and a constant vorticity distribution was obtained in [16]. We use the general form of the expression for the self-induced velocity of both vortex filaments

$$
\begin{aligned}
u_{b}(x)=\frac{\Gamma_{x}}{4 \pi x}\left\{\frac{x^{2}}{l^{2}+x^{2}}\left[\ln \frac{x}{\varepsilon}-\frac{1}{4}-\frac{3}{2} \ln \frac{x l}{l^{2}+x^{2}}+2+\frac{l^{2}}{x^{2}}-\frac{\sqrt{l^{2}+x^{2}}\left(x^{2}+3 l^{2}\right)}{l x^{2}}\right]+\right. \\
\left.=+\frac{x^{6} l^{2}}{\left(l^{2}+x^{2}\right)^{4}}\left[\left(\frac{l^{4}}{x^{4}}-3 \frac{l^{2}}{x^{2}}+\frac{3}{8}\right) \zeta^{*}-\frac{27}{8}+2 \frac{l^{4}}{x^{4}}+\frac{x^{2}}{l^{2}}\right]+4 \frac{x \sqrt{l^{2}+x^{2}}}{l^{2}} I_{1}\left(\frac{x}{l}\right) K_{1}^{\prime}\left(\frac{x}{l}\right)\right\}+o((13))
\end{aligned}
$$

for the vortex position at the point $x=a$ or $x=b$ and circulation intensity $\Gamma_{a}$ or $\Gamma_{b}$, respectively $\left(I_{1}\right.$ and $K_{1}$ are the modified Bessel functions; the prime is used to indicate their derivatives; $\zeta^{*}=1.20206$ ). Formula (3) takes into account the correction for the finite size of the helical vortex with a uniform distribution of vorticity in the form of the term $1 / 4$ in the first square brackets [16].

The expression for the projection of the circumferential velocity $V_{I n d}^{(y)}(x)$ induced at the point $x$ by the vortex located at the point $y$ in the cross section $z=0$ with allowance for the correction for vertical displacement is written as

$$
V_{\text {Ind }}^{(y)}(x) \equiv w_{\chi}(x ; y)=u_{\theta}(x ; y)-u_{z}(x ; y) x / l,
$$

where $u_{z}(x ; y)$ and $u_{\theta}(x ; y)$ are the axial and azimuthal velocities in the motionless coordinate system induced at the point $x$ by the vortex located at the point $y$ at $\theta=0$. As it was expected, the expression for velocity (4) coincides with the expression for the projection of velocity in the helical coordinate system for the corresponding variable $\chi=\theta-z / l[6]$. Neglecting the influence of the finite size of the vortex core on the field induced at the points of the neighboring vortex, the expression for this velocity "induced" on the other vortex can be written in the following form [15]:

$$
w_{\chi}(x, y, \chi)=\frac{\Gamma}{2 \pi}\left\{\begin{array}{c}
-x / l^{2} \\
1 / x
\end{array}\right\}+\frac{\Gamma y}{\pi l^{2}}\left(\frac{l}{x}+\frac{x}{l}\right) \operatorname{Re}\left\{\begin{array}{c}
H_{1}^{0,1}(x / l, y / l, \chi), \ldots x \leqslant y \\
H_{1}^{1,0}(y / l, x / l, \chi), \ldots y<x
\end{array}\right\} .
$$

The Kapteyn-type series in Eq. (5)

$$
H_{M}^{I, J}(x, y, \chi)=\sum_{n=1}^{\infty} m^{M} I_{m}^{\langle I\rangle}\left(\frac{m x}{l}\right) K_{m}^{\langle J\rangle}\left(\frac{m y}{l}\right) \mathrm{e}^{i m \chi}
$$

are expressed via the modified Bessel functions $I_{m}^{\langle 0\rangle}(m x / l)$ and $K_{m}^{\langle 0\rangle}(m y / l)$ ad their derivatives $I_{m}^{\langle 1\rangle}(m x / l)$ and $K_{m}^{\langle 1\rangle}(m y / l)$ at $x \leqslant y$. These series can be contracted into a closed finite form [16], which can simplify the calculations, especially in the limiting cases, e.g., if the filaments move closer to each other.

\section{RESULTS OF INVESTIGATING UNIFORM ROTATION OF THE HELICAL VORTEX PAIR}

For joint uniform rotation of the vortex pair, it is sufficient to require the coincidence of the angular velocities of both vortices:

$$
\Omega_{0} \equiv \Omega^{(a)}=\Omega^{(b)}
$$

i.e., using formulas (2)-(5), one has to find the total circumferential velocities of vortex motion in a fixed cross section $z=0$. As it follows from Fig. 1a, in the case of vortices with a straight-line axis $(h=\infty)$, this is possible only for different values of circulation intensity, when the condition $\Gamma_{a} / \Gamma_{b}=-b / a$ is satisfied. Let us analyze how the motion of helical vortices with a finite value of $h=2 \pi l$ changes and whether their uniform rotation (7) at $\Gamma_{a}=-\Gamma_{b}$ in the presence of additional velocity of self-induced motion caused by vortex filament curvature is possible. It can be assumed that the induced velocities remain almost unchanged as compared to the case of point vortices (see Fig. 1a) because they mainly depend on the distance between the points $a$ and $b$ and on the absolute value of their circulations $\Gamma_{a}$ and $\Gamma_{b}$ in a large range of their helix pitch $h$. Therefore, for simplification, the velocities 


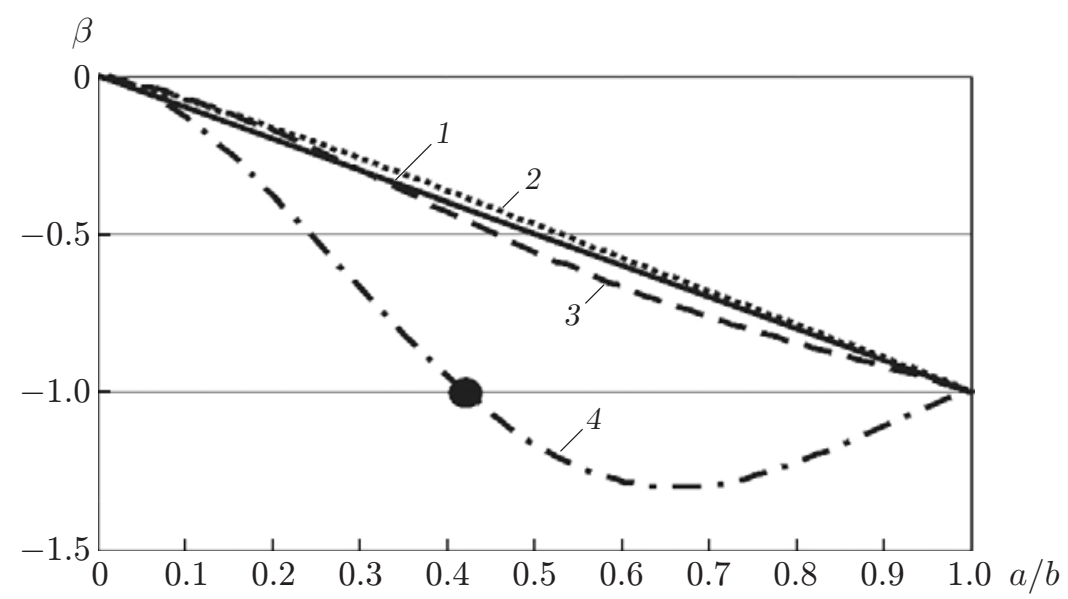

Fig. 3. Ratio of the circulation intensities $\beta$ versus the ratio $a / b$ in the case of equilibrium rotation of vortices for $\varepsilon=0.05$ and different values of the helix pitch: $h=\infty(1), 5(2), 2(3)$, and $1.5(4)$; the point is the solution for identical absolute values of circulation intensities with the opposite signs.

(a)

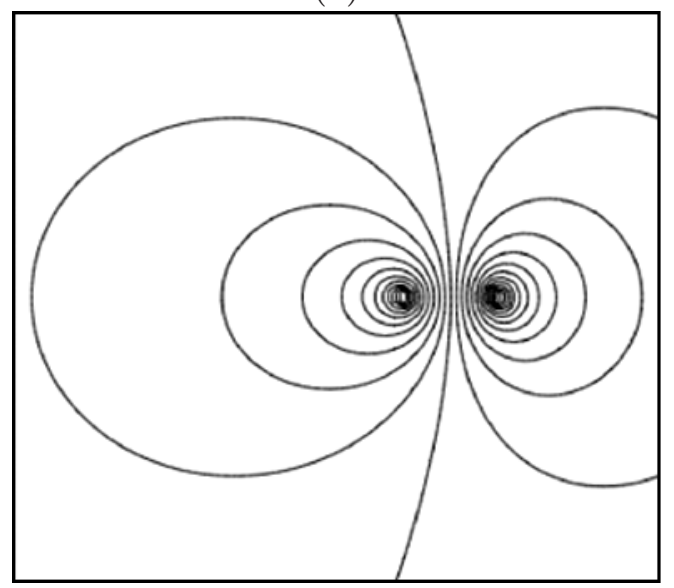

(b)

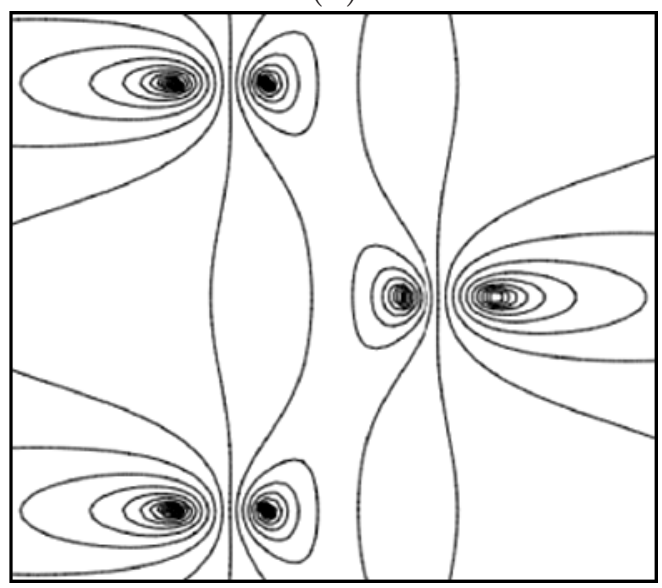

Fig. 4. Horizontal (a) and meridional (b) cross sections of the stream tubes in the case of motion of the helical vortex pair shifted away from the center of rotation.

in Fig. 2 are approximately identical $\left(V_{I n d} \approx V_{I n d}^{(b)}(a) \approx V_{I n d}^{(a)}(b)\right)$ to indicate that this component of the velocity field cannot rapidly balance the ratio $b / a$ for identical absolute values of the circulation intensities. At the same time, the presence of the self-induced component in the case with different signs of the circulation intensities leads to rotation of the vortices in the opposite directions, which can be sufficient for uniform rotation of the vortex pair.

To verify this assumption, we introduce the ratio of the circulation intensities $\beta=\Gamma_{b} / \Gamma_{a}$. By varying the ratio $a / b$ determining the vortex positions in the pair and taking into account substitution of (1)-(6) for several fixed values of the helix pitch $h$, we find the values of $\beta$ for which equality (7) is satisfied.

Figure 3 shows the ratio of circulation intensities $\beta$ as a function of the ratio $a / b$. The linear dependence 1 correspond to the Helmholtz solution for point vortices at $\Gamma_{b} / \Gamma_{a}=-a / b$. It follows from this dependence that there are no finite distances between the points $a$ and $b$ for which the circulation intensities have identical values. Nevertheless, such a solution with identical values of circulation intensities and opposite signs does exit for the helical vortex (see the point in Fig. 3). It is seen in Fig. 3 that equilibrium rotation arises at the helix pitch 
value $h<2$ and fixes the unequivocal positions of vortices in the pair $(a / b \approx 0.4, h=1.5)$. This result offers an explanation for the existence of equilibrium configurations of helical vortices behind rotating wings and blades observed in scarce calculations and experiments $[12,15]$. This cannot be explained by using the solution for the jointly rotating pair of point vortices because the self-induced motion described by Eq. (3) is absent in this case. Examples of streamlines for the helical vortex pair denoted by the point in Fig. 3 are presented in Fig. 4 in two diametral cross sections. Figure $4 \mathrm{~b}$ (meridional cross section) shows the cross sections of the stream tubes for the case of motion of the helical vortex pair shifted away from the center of rotation by the distance $a \approx 0.4$ for $b=1$, while Fig. 4a shows the corresponding horizontal cross sections.

\section{CONCLUSIONS}

The results of the present theoretical study confirm the existence of a uniformly rotating pair of helical vortices with identical absolute values of circulation intensities of the opposite signs for certain values of the helix pitch. The existence of such equilibrium pairs of helical vortices is important for further development of vortex dynamics and also for solving applied problems and designing blades in turbomachinery with predictable evolution of rotor vortex wakes.

This work was performed within the framework of the State Program supporting research performed under the guidance of leading scientists in Russian institutes of higher education (Agreement No. 075-15-2019-1923).

\section{REFERENCES}

1. S. V. Alekseenko, S. I. Shtork, "Experimental observation of vortex filament interaction," JETP Lett. 59, 775-780 (1994).

2. H. Aref, J. B. Kadtke, I. Zawadzki, et al., "Point Vortex Dynamics: Recent Results and Open Problems," Fluid Dynamic Res. 3, 63-74 (1988).

3. J. J. Thomson, A Treatise on the Motion of Vortex Rings (L.: Macmillan, 1883).

4. T. H. Havelock, "The Stability of Motion of Rectilinear Vortices in Ring Formation," Philos. Mag., No. 11, 617-633 (1931).

5. W. Thomson, (Lord Kelvin). "Floating Magnets (Illustrating Vortex-Systems)," Nature, No. 18, 13-14 (1878).

6. V. L. Okulov "On the Stability of Multiple Helical Vortices," J. Fluid Mech. 521, 319-342 (2004). DOI: $10.1017 / \mathrm{s} 0022112004001934$.

7. I. Delbende, B. Piton, M. Rossi, "Merging of Two Helical Vortices," Europ. J. Mech. B. Fluids 49, 363-372 (2015).

8. C. Selçuk, I. Delbende, M. Rossi, "Helical Vortices: Quasiequilibrium States and their Time Evolution," Phys. Rev. Fluids, No. 2, 084701 (2017).

9. V. L. Okulov, "An Acentric Rotation of Two Helical Vortices of the Same Circulations," Regular Chaotic Dynamics 21 (3), 267-273 (2016).

10. H. von Helmholtz, "Über Integrale der Hydrodynamischen Gleichungen, Welche den Wirbelbewegungen Entsprechen," J. Reine Angew. Math. 55, 25-55 (1858).

11. S. V. Alekseenko, Theory of Concentrated Vortices: An Introduction Eds. by S. V. Alekseenko, P. A. Kuibin, V. L. Okulov. (Berlin: Springer-Verlag, 2007).

12. S. Salunkhe, O. El Fajri O, S. Bhushan, et al., "Validation of Tidal Stream Turbine Wake Predictions and Analysis of Wake Recovery Mechanism," J. Marine Sci. Engng. 7, 362-386 (2019).

13. N. E. Joukowsky, "Vortex Theory of the Marine Propeller I," Trudy Otd. Fiz. Nauk Obshchestva Lubitelei Estestvoznaniya 16 (14), 1-47 (1912).

14. V. L. Okulov, J. N. Sørensen, D. H. Wood, "The Rotor Theories by Professor Joukowsky: Vortex Theories," Progr. Aerospace Sci. 73, 19-46 (2015). DOI: 10.1016/j.paerosci.2014.10.002.

15. H. U. Quaranta, H. Bolnot, T. Leweke, "Long-Wave Instability of a Helical Vortex," J. Fluid Mech. 780, $687-716(2015)$.

16. V. L. Okulov, J. N. Sørensen, "The Self-Induced Motion of a Helical Vortex," J. Fluid Mech. 883, A-5 (2020). 
17. R. L. Ricca, "The Contributions of Da Rios and Levi-Civita to Asymptotic Potential Theory and Vortex Filament Dynamics," Fluid Dynamics Res. 18, 245-268 (1996).

18. Y. Fukumoto, V. L. Okulov, D. H. Wood, "The Contribution of Kawada to the Analytical Solution for the Velocity Induced by a Helical Vortex Filament," Appl. Mech. Rev. 67 (6), 060801 (2015). DOI: 10.1115/1.4031964.

19. V. L. Okulov, E. S. Gesheva, P. A. Kuibin, et al., "Difference in the Motion of a Helical Vortex and the Motion of Particles along the Vortex Axis," Teplofiz. Aeromekh. (2020) (in press).

20. P. G. Saffman, Vortex Dynamics. Cambridge (Cambridge Univ. Press, 1992).

21. R. L. Ricca, "The Effect of Torsion on the Motion of a Helical Vortex Filament," J. Fluid Mech. 273, 241-259 (1994).

22. J. Boersma, D. H. Wood, "On the Self-Induced Motion of a Helical Vortex," J. Fluid Mech. 384, 263-280 (1999).

23. P. A. Kuibin, V. L. Okulov, "Self-Induced Motion and Asymptotic Expansion of the Velocity Field in the Vicinity of a Helical Vortex Filament," Phys. Fluids 10, 607-614 (1998). 